found in partial or complete androgen insensitivity syndrome. The aim of this study was to determine whether even the most minor forms of isolated hypospadias are associated with AR mutations and thus whether all types of hypospadias warrant molecular analysis of the AR.

Methods 292 Caucasian children presenting with isolated hypospadias (no micropenis, no cryptorchidism) and 345 controls were included prospectively. Mutational analysis of the AR through direct sequencing (exons 1-8) was performed.

Results Five missense mutations of the AR were identified in 9 patients with glandular or penile anterior $(n=5)$, penile midshaft $(n=2)$ and penile posterior $(n=2)$ hypospadias, i.e., 3\%: p.Q58L (c.1288 A>T), 4 cases of p.P392S (c.2289 C>T), 2 cases of p.A475V (c.2539 C>T), p.D551H (c.1651 G>C) and p.Q799E (c.3510 C>G). None of these mutations was present in the control group. Four mutations are novel findings since 1 has never been reported to date (p.D551H) and 3 have never been reported in patients with genital malformation but only in isolated infertility: p.Q58L, p.P392S, and p.A475V. It is notable that micropenis, a cardinal sign of AIS, was not present in any patients.

Conclusion AR mutations may play a role in the cause of isolated hypospadias, even in the most minor forms. Identification of this underlying genetic alteration is important for proper diagnosis and may significantly improve the follow-up of these patients during puberty, especially regarding future fertility.

\section{THE FEASIBILITY OF TISSUE EXPANDERS IN RECONSTRUCTION OF GIANT CONGENITAL MELANOCYTIC NEVI IN CHILDREN}

doi:10.1136/archdischild-2012-302724.1587

'M Fahmy, ${ }^{2}$ AA Mazy. 'Pediatric Surgery, Al Azher University, Cairo; ${ }^{2}$ Anesthesia, Al Masuera University, Masuera, Egypt

Background Children with Giant Congenital Melanocytic Nevi (GCMN) carry a great challenge to the pediatric and reconstructive surgeons to cover the widely exposed area after its excision. A variety of treatment options exists for the management of such cases. In this retrospective review of selected group of children had a GCMN of their abdominal walls were managed with implantation of tissue expanders (TE) for staged reconstruction, patients evaluated with respect to complications, general and esthetic patient and parents satisfaction.

Objective Our purpose was to study the feasibility of use tissue expanders in the management of children who had GCMN with special emphasis to the complications and children and their parents satisfaction.

Material and Methods Retrospective data collection of the 12 patient's charts, operative data of 86 surgical procedures and follow up visits were calculated and analyzed, with. Statistical analysis done using the Student $t$ test, and $\mathrm{P}<0.05$ was considered statistically significant.

Results In a period of 4 years, from 2004 to 2008, the results of using 37 different sizes tissue expanders and 86 operative procedures in a 12 child, 9 boys and 3girls aged from 2 to 12 years with different types of abdominal wall GCMN will be discussed.

Conclusion Tissue expander is a useful and feasible tool for reconstruction of the abdominal wall in cases of GCMN in children. Parents and children satisfaction and body image are generally acceptable.

\section{COMPARISON OF CIRCUMCISIONS PERFORMED WITH PLASTIC CLAMP AND CONVENTIONAL DISSECTION TECHNIQUE}

doi:10.1136/archdischild-2012-302724.1588
FM Senel, F Misirlioglu. Dr. Sami Ulus Hospital, Ankara, Turkey

Background and Aims In the current study we serched the outcome of circumcisions performed with a new plastic clamp. The results were compared to those who underwent conventional circumcision.

Methods We analyzed the short-term complications of the procedure among the 3650 children who were consecutively circumcised by plastic Alis klamp technique. The results were compared to those of 1275 children who were circumcised by conventional technique. Results The rate of infection $(0.4 \%)$ and bleeding $(0.2 \%)$ after plastic clamp were significantly lower compared to those children circumcised by conventional technique (3.2\% and $4.1 \%$; $<0.0001$ ). Buried penis occured in similar rates among both groups. We did not encounter any penile injury in either groups. The total complication rates of plastic clamp and conventional techniques were found as $1.8 \%$ and $8.7 \%$, respectively $(p<0.0001)$. The mean duration of circumcision time with plastic clamp and conventional technique was $4.1 \pm 1.2$ min and $24 \pm 3.6$ min, respectively ( $<<0.0001$ ).

Conclusion Plastic Alis klamp technique is suggested as the circumcision procedure of choice due to reduced complications, as well as short duration and ease of application.

Abstract 1588 Table 1 Comparison of complications

\begin{tabular}{lllllc}
\hline & Plastic clamp (n 3650) & \% & Conventional (n 1275) & $\%$ & p \\
\hline Age (years) & $6.2 \pm 1.5$ & & $6.9 \pm 1.8$ & & \\
Infection & 14 & 0.4 & 41 & $3.2<0.0001$ \\
Bleeding & 7 & 0.2 & 52 & $4.1<0.0001$ \\
Buried penis & 44 & 1.2 & 18 & 1.4 & NS \\
Duration (min) & $4.1 \pm 1.2$ & & $24 \pm 3.6$ & & $<0.0001$ \\
Total complications & 65 & 1.8 & 111 & $8.7<0.0001$ \\
\hline
\end{tabular}

\section{ROLE OF THORACOSCOPY IN THE TREATMENT OF LOCULATED PLEURAL EMPYEMA IN PEDIATRIC PATIENTS: ABOUT 73 CASES}

doi:10.1136/archdischild-2012-302724.1589

${ }^{1} \mathrm{~J}$ Chahed, ${ }^{2} \mathrm{~K}$ Maazoun, ${ }^{2} \mathrm{~S}$ Hidouri, ${ }^{2} \mathrm{~A}$ Ksia, ${ }^{2} \mathrm{~K}$ Krichene, ${ }^{2} \mathrm{~L}$ Sahnoun, ${ }^{2} \mathrm{M}$ Mekki, ${ }^{2}$ A Nouri. ${ }^{1}$ Pediatric Surgery; ${ }^{2}$ EPS Fattouma Bourguiba, Monastir, Tunisia

Objective Evaluate the results of thoracoscopy for the treatment of located pleural empyema.

Methods Retrospective study of 73 patients with loculated pleural empyema in pediatric surgery department of Monastir during the period between 1997-2010. Located empyema was confirmed in all patients through imaging (ultrasonography or tomography of chest).

Results : The age of patients ranged between 2 months- 16 years (mean age: 41.5 months) with a sex ration of 1.28. Fever was present in $98 \%$ of the cases. 25 patients had severe respiratory distress and mechanical ventilation was necessary in 4 cases. Bacteriology of the pleural liquid was positive in 12 cases. All patients had intravenous antibiotherapy during a mean period of 20 days. Sixteen patients $(22 \%)$ were treated by thoracic drainage. Intrapleural fibrolysis was indicated in 5 cases (7\%). One patient had an open thoracotomy for lung abscess with a loculated pleural empyema. Thoracoscopy was performed in 51 cases (70\%). It was indicated after thoracic drainage failure in 14 cases. It was indicated for firstline before any prior thoracic drainage in 37 cases because of a multiloculated aspect at ultrasonography.

Conclusion Management of pleural empyema is still controversal in children and surgical indication is often delayed. Early first-line thoracoscoy yields a better clinical outcome for pediaric patients with pleural loculated empyema, with apparent decreased morbidity, earlier 